\title{
Modulation of CYP3A4 by the RGD- and Neurotensin(8-13)-analogues
}

Kaloyan Georgiev ${ }^{1}$, Maya Radeva ${ }^{1}$, Anelia Balacheva ${ }^{2}$, Sylvia Michaylova ${ }^{1}$, Tamara Pajpanova ${ }^{2}$

${ }^{1}$ Medical University of Varna, Bulgaria

${ }^{2}$ Bulgarian Academy of Sciences, Bulgaria

https://doi.org/10.17952/35EPS.2018.215

\section{Introduction}

RGD- and Neurotensin(8-13)-based drug design is a hopeful perspective for drug development [1,2]. There is a great deal of data of the observed therapeutic effects of the both sequences (e.g. anticancer, analgesic etc.), but little is known about the pharmacokinetic of these peptides and the possible drug interactions in which they can be involved. Because of that, we decided to check whether these sequences are able to affect the activity of the most common cytochrome P450 enzyme CYP3A4, responsible for the most drug interactions.

\section{Materials and Methods}

Peptide synthesis and analysis

Peptides in this study were synthesized by manual solid-phase procedures using techniques for Fmoc-protected amino acids on Wang or MBHA Rink-Amide peptide resins respectively. 20\% Piperidine in DMF was used for deprotection of Fmoc-groups and DIC and HBTU were employed as a coupling agent. Simultaneous deprotection and cleavage from the resin was accomplished by treatment with TFA/TIS/ water (95:2.5:2.5) for $3 \mathrm{~h}$ at room temperature. Crude peptides were purified by preparative TLC and their purity was checked by analytical HPLC.

Measurement of CYP3A4 activity in vitro

The test compound was mixed with a master pre-mix comprising CYP450 BACULOSOMES ${ }^{\circledR}$ reagent and regeneration system, which contained glucose-6-phosphate and glucose-6-phosphate dehydrogenase. The mixture was incubated at room temperature for $20 \mathrm{~min}$. Following incubation, CYP enzyme-specific substrate (Vivid DBOMF for CYP3A4) and NADP+ were added and the mixture was incubated at room temperature for $30 \mathrm{~min}$. CYP activity was evaluated by measuring the fluorescence of the fluorescent metabolite generated from CYP3A4 enzyme-specific substrate. The fluorescence was measured using a microplate reader BioTek Synergy.

\section{Results and Discussion}

We used two RGD-analogues modified in the first position with nonproteinogenic amino acids - Agb (S-2-amino-3-guanidino-butyric acid) (1, AgbGD) and Agp (S-2-amino-3-guanidino-proponic acid) (2, AgpGD), Neurotensin (8-13) (3, Arg-Arg-Pro-Tyr-Ile-Leu) and modified at position eight and nine with Lys-Cav (4, Lys-Cav-Pro-Tyr-Ile-Leu). We used the fourth peptides at concentrations of 25 to $100 \mu \mathrm{M}$.

Both RGD-analogues ( 1 and 2) showed minor modification in the activity of CYP3A4 (Figure 1). Only with the largest used concentration, $100 \mu \mathrm{M}$, the effect was pronounced with inhibition of $50 \%$ of the activity of enzyme. 


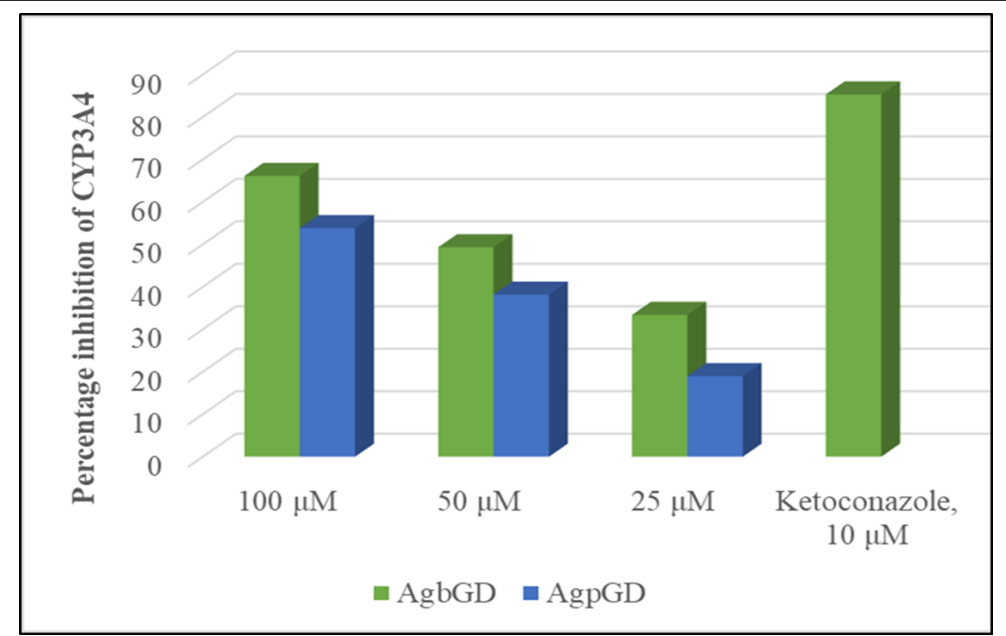

Figure 1: Modulation of CYP3A4 by the RGD analogues - AgbGD and AgpGD.

Neurotensin(8-13) (3) and its analogue (4) did not show activity on CYP3A4 (Figure 2). All three concentrations modulate insignificantly CYP3A4 enzyme.

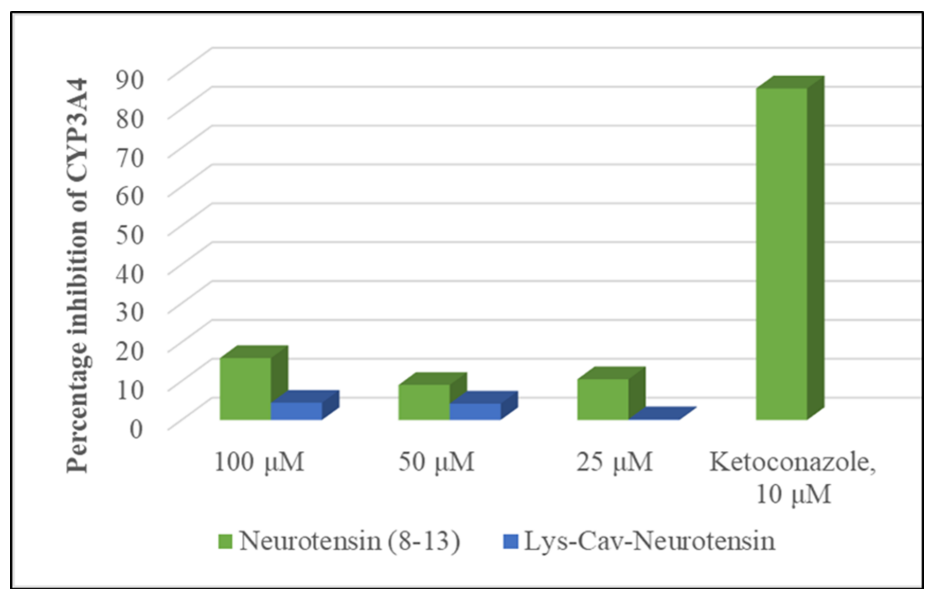

Figure 2: Modulation of CYP3A4 by the Neurotensin (8-13) and its analogue Lys-Cav-Neurpotensin

\section{Conclusion}

The results have shown that the use of the RGD-analogues ( 1 and 2) should be monitored for possible drug interactions, while using Neurotensin(8-13)-sequences does not endanger drug interactions at the cytochrome P450 3A4 level.

\section{Acknowledgements}

The study was supported by National Science Fund, Ministry of Education and Science, Bulgaria; Project: DN19/17 and by Fund Science, at the Medical University of Varna/Bulgaria; Project: 16021

\section{References}

1. Georgiev, K., Balacheva, A., Peycheva, E., Georgieva, M., Dzimbova, T., Georgiev, L., Iliev, I., Detcheva, R., Miloshev, G., Pajpanova T. In: Kokotos, G., Constantinou-Kokotou, V., Matsoukas, G. (eds) Peptides 2012. Proceedings of the 32st European Peptide Symposium, Athens, Greece, p. 368-369. ISBN: 978-960-466-121-3.

2. Dzimbova, T., Stoeva, S., Tancheva, L., Georgieva, A., Kalfin, R., Pajpanova, T. In: Naydenova, E., Danalev, D, Pajpanova, T (eds) Peptides 2014. Proceedings of the 33rd European Peptide Symposium, Sofia, Bulg aria, $\mathrm{p}$ 260-261. ISBN: 978-619-90427-1-7. 